\title{
Validation of the Italian Yale Food Addiction Scale in postgraduate university students
}

\author{
Gian Mauro Manzoni ${ }^{1,2} \mathbb{D}$. Alessandro Rossi ${ }^{1,4} \cdot$ Giada Pietrabissa $^{1,3} \cdot$ Giorgia Varallo $^{1} \cdot$ Enrico Molinari ${ }^{1,3}$. \\ Eleonora Poggiogalle ${ }^{5}$. Lorenzo Maria Donini ${ }^{5}$. Giulietta Tarrini ${ }^{6} \cdot$ Nazario Melchionda $^{6}$. Carla Piccione ${ }^{7}$. \\ Giovanni Gravina ${ }^{8}$. Gianluigi Luxardi ${ }^{9}$. Emilia Manzato ${ }^{10}$. Romana Schumann ${ }^{11}$. Marco Innamorati ${ }^{12}$. \\ Claudio Imperatori ${ }^{12} \cdot$ Mariantonietta Fabbricatore $^{12} \cdot$ Gianluca Castelnuovo $^{1,3}$
}

Received: 18 November 2017 / Revised: 22 February 2018 / Accepted: 23 February 2018 / Published online: 12 March 2018

๑) Springer International Publishing AG, part of Springer Nature 2018

\begin{abstract}
Purpose This study was aimed to examine the structural and construct validity of the Italian version of the Yale Food Addiction Scale in a multisite sample of postgraduate students.

Methods Two hundred and fifty-six subjects $(78.1 \%$ females) aged from 18 to 53 years $($ mean $=23.93, \mathrm{SD}=4.96)$ and attending different postgraduate university programs at multiple Italian universities completed the Italian YFAS, the Italian Binge Eating Scale (BES), the Italian Eating Attitudes Test-26 and the Italian Dutch Eating Behavior Questionnaire (DEBQ) online through Qualtrics.

Results Confirmatory Factor Analysis showed that the single-factor model of the Italian YFAS including all original items had adequate fit indexes $\left(\chi^{2} 252=454.183 ; p<0.001\right.$; normed $\chi^{2}=1.802$; RMSEA $=0.056 ; 90 \%$ CI 0.048-0.076; CFI $=0.761$; WRMR = 1.592). However, item analysis revealed that item\#25 had zero variance (all subjects were assigned the same score after item dichotomization) and item\#24 had a low factor loading, and were thus removed. Furthermore, item\#10 and item\#11 showed to be almost perfectly correlated $(r=0.998)$ and were thus parceled. The resulting 19-item single-factor model revealed a better fit to the data $\left(\chi^{2} 152=235.69 ; p<0.001\right.$; normed $\chi^{2}=1.556$; RMSEA $=0.046$; 90\% CI 0.034-0.058; $\mathrm{CFI}=0.858$; WRMR $=1.236)$ and its internal consistency was acceptable $(\mathrm{KR}-20=0.72)$. Also, a single-factor model including the seven diagnostic symptoms was tested and showed adequate fit values $\left(\chi^{2} 20=41.911 ; p<0.003\right.$; normed $\chi^{2}=2.09$; RMSEA $=0.065 ; 90 \%$ CI 0.037-0.093; CFI =0.946; WRMR =1.132). Statistically significant and small-to-high correlations were found with all convergent measures, in particular with the BES.

Conclusion The Italian 19-item YFAS resulted to be a valid and reliable tool for the assessment of food addiction in postgraduate students.

Level of evidence Level V, descriptive study.
\end{abstract}

Keywords Food addiction · YFAS · Validity · University students

\section{Introduction}

Over recent years, the clinical and scientific perspectives on addictive disorders have extended to include excessive and repetitive behavioral patterns that appear comparable to substance use disorders $[1,2]$. In addition to gambling,

This article is part of the topical collection on Food Addiction.

Gian Mauro Manzoni

gm.manzoni@auxologico.it

Extended author information available on the last page of the article internet gaming, sex addiction, exercise addiction and shopping addiction [3], food addiction (FA) has also gained attention. It was defined as an abnormal pattern of overconsumption of food in association with symptoms descriptively similar to those of substance dependence, i.e., loss of control, tolerance, withdrawal and impulsivity [4], and has been investigated as a new etiological hypothesis underlying obesity [5-8]. Several neurobiological parallelisms have been observed between food addiction and substance use disorder [9-12]. For example, neuronal circuits modulated by dopamine were shown to be activated by both drugs and hyper-palatable foods, and both conditions resulted to 
exhibit both a reduced availability of dopamine D2 receptors and a decreased metabolism in prefrontal regions involved in inhibitory control [13-15].

One of the most used self-report measures of FA is the Yale Food Addiction Scale (YFAS). It was originally developed on the basis of the DSM-IV-TR substance dependence criteria [4] and, even though the changes of the substance use disorder in the DSM 5, but, given the overlap with the DSM- 5 criteria for the substance use disorder, it can still be applied [5, 6].

The YFAS was firstly validated in a non-clinical sample $(N=353)$ of undergraduate college students [4] and demonstrated both a single-factor structure and an adequate internal consistency $(\alpha=0.86)$. To support its construct validity, Gearhardt et al. [16] used a sample of lean to obese women and found out that higher scores on the YFAS symptoms count were associated with patterns of neuronal activation similar to those observed in drug addiction, i.e., elevated neural activation of the reward circuitry in response to anticipated food intake.

The psychometric properties of the YFAS were later assessed in a sample of obese adults $(N=81)$ with binge eating disorder and the single-factor structure was further confirmed [17].

Meule et al. [18] made available the German version of the YFAS and assessed its psychometric proprieties in a study involving non-clinical subjects. A one-factor structure was still found and internal consistency, as well as convergent and divergent correlations, resulted to be comparable to the ones reported for the original version [4]. Both the factor structure and the internal consistency of the German YFAS were further replicated in a sample of obese candidates $(N=96)$ for bariatric surgery [19].

Like the original and the German versions, the French translation of the YFAS was also first validated in a sample of non-clinical subjects $(N=553)$ [20]. The one-factor structure explained a high proportion of variance, which is consistent with the original results [4], and the internal consistency of both the symptoms count and the diagnostic scores were replicated. Furthermore, a high convergent validity with measures of binge eating was showed [20].

The Spanish version of YFAS was developed and validated by Granero et al. [21] in a merged sample $(N=207)$ of adults with an eating disorder $(N=125)$ and non-clinical controls $(N=82)$. Results showed a good internal structure of the one-dimensional solution $(\alpha=0.95)$ and suggested that the YFAS discriminates between individuals with an eating disorder and healthy controls.

Chen et al. [22] developed the Chinese version of the YFAS and tested its psychometric properties in a sample of female normal school students $(N=950)$. Internal consistency resulted to be adequate for both the symptoms count and the diagnostic scores, which correlated significantly with the Eating Attitudes Test and the Binge Eating Scale. On the contrary, no or small correlations were found with the Behavioral Inhibition System/Behavioral Activation System and the Regulatory Emotional Self-efficacy [22].

Innamorati et al. [23] translated the YFAS into Italian language and investigated its psychometric properties in a merged sample of overweight/obese patients $(N=300)$ and healthy controls $(N=300)$. The one-factor model did not fit the data and the analysis of items supported the development of a new 16-item version of the questionnaire. The YFAS16 showed a satisfactory internal consistency $(\alpha=0.85)$ and good discriminant as well as concurrent validity [23].

The aim of the current study was to examine some psychometric properties of the full-length Italian version of the YFAS in a sample of postgraduate students. In particular, we sought to assess its structural validity, internal consistency and convergent validity.

\section{Methods}

\section{Sample and procedure}

Participants were 256 postgraduate university students (78.9\% of females; one did not indicate his/her own gender on the demographic form) aged from 18 to 53 years (mean $=23.87, \mathrm{SD}=4.98)$ and attending different postgraduate university programs at multiple Italian universities. They were enrolled during classes by academic teachers who are members of the Italian Society for the study of Eating Disorders (SIS-DCA) and who were invited to collaborate to the study at the VIII annual meeting of the Society.

Students who accepted to participate and provided their e-mail addresses were sent an e-mail including a Qualtrics link to the online questionnaires, which were administered after obtaining their digital informed consent.

\section{Measures}

Participants completed first a demographic form (gender, age and BMI) and were then asked to fill in the following self-report questionnaires.

\section{YFAS}

The Italian translation of the YFAS that was used in the present study was obtained by Innamorati et al. [23] and can be requested to the corresponding author. It consists of 25 items originally developed by Gearhardt et al. [4] according to the DSM-IV-TR substance dependence symptoms criteria, as operationalized in the Structured Clinical Interview for DSM-IV Axis I Disorders [24]. Specifically, the YFAS investigates: (A) "substance taken in larger amount and 
for longer period than intended"; (B) "persistent desire or unsuccessful attempts to quit"; (C) "much time/activity to obtain, use, recover"; (D) "activities given up or reduced", (E) "use continues despite knowledge of adverse consequences"; (F) "tolerance"; (G) "withdrawal symptoms and substance taken to relieve withdrawal"; $(\mathrm{H})$ "impairment or distress". Twenty items have a 5-point Likert response scale (three out of them-item\#17, item \#18 and item \#23-are primers), while eight items (from item \#17 to item \#24) are dichotomous. The YFAS has two scoring options: (a) a continuous score-symptom count-that indicates the number of food addiction symptoms that have been met and (b) a diagnostic score which provides a diagnosis of food addiction when the subject presents at least three symptoms and reports clinically significant impairment and/or distress [4].

\section{BES}

The Binge Eating Scale (BES), originally developed by Gormally et al. [25] and translated into Italian language by Ricca et al. [26], measures binge eating severity by investigating the frequency of thoughts, feelings and behaviors associated with Binge Eating Disorder. It consists of 16 items that are groups of three or four statements increasing in severity. For each item, subjects have to choose the statement that best describes his/her condition. The original BES was reported to have satisfactory internal consistency and to discriminate well between clinical and non-clinical individuals [25], showing also an extremely high concordance with the interview-based diagnosis of Binge Eating Disorder [27]. Several studies explored its factorial structure and found two different components (feelings/cognitive and behavioral) [25, 28-31]. Marek et al. [29] tested also a bi-factor model, which showed a better fit to the data than the two-factor model. This result was further confirmed by Imperatori et al. (2015), who assessed the factorial structure of the Italian version of the BES in a large sample of overweight patients. In this study, Cronbach's alphas were $0.870,0.784$ and 0.774 for the BES Total scale, the Cognitions/feelings scale and the Behaviors scale, respectively.

\section{EAT-26}

The Eating Attitudes Test-26 (EAT-26), originally developed by Garner et al. [32] and translated into Italian language by Dotti and Lazzari [33], is a measure of core symptoms, worries and usual behaviors concerning eating disturbances and/ or eating disorders (ED). It consists of 26 items that compose 3 different subscales: "dieting" (avoidance of fatty foods and preoccupation with losing weight), "bulimia" (bulimic tendencies) and "oral control" (food intake mode and its control). Items are rated on a six-point Likert scale ranging from "never" to "always", where higher scores suggest a more severe symptomatology [32, 33]. An oblique three-factor solution was found for both the original version [32] and the Italian translation [33], which showed also high internal and test-retest reliabilities. Furthermore, the Italian EAT-26 showed good convergent, discriminant and criterion-related validity with other measures of ED symptomatology [33]. In this study, Cronbach's alphas were 0.857, 0.854, 0.609 and 0.551 for the total scale, the Dieting scale, the Bulimia scale and the Oral Control scale, respectively.

\section{DEBQ}

The Dutch Eating Behavior Questionnaire (DEBQ), originally developed by Van Strien et al. [34] and translated into Italian language by Dakanalis et al. [35], is composed of 33 items rated on a 5-point Likert scale (ranging from "never" to "very often") and grouped into 3 subscales: "emotional eating" (13 items), "external eating" (10 items) and "restrained eating" (10 items). The "Emotional eating" scale measures the tendency to use food as a way of coping with psychological problems and/or to alleviate the subject from distress; the "external eating" scale measures the frequency of eating in response to external stimuli such as the sight and smell of food; finally, the "restrained eating" scale measures the frequencies of restrictive conscious behavior of food intake [34]. Both the original and the Italian versions were reported to have a strong three-factor structure, high internal consistency and high test-retest reliability after a 4-week period [34-37]. Moreover, the Italian DEBQ proved to be invariant across sex, BMI and age [35]. In this study, Cronbach's alphas were $0.927,0.925,0.881$ and 0.737 for the total scale, the Restrained Eating scale, the Emotional Eating scale and the External Eating scale, respectively.

\section{Statistical analysis}

All questionnaires were first scored according to their respective scoring procedures. With respect to the YFAS, item\#1 to item\#16 and item \#25 were dichotomized using the thresholds proposed by Gearhardt et al. in their first article [4]. After dichotomization, item\#25 (How many times in the past year did you try to cut down or stop eating certain foods altogether?) resulted to have zero variance, because all participants were assigned the same score (zero) and was thus excluded from all the following analyses. All items' descriptive statistics after dichotomization are reported in Table 1.

The factorial structure of the Italian YFAS was then assessed by means of Confirmatory Factor Analysis (CFA). The original model including also the item primers (item\#17, item\#18, item\#23) was tested first. After evaluating their factor loadings, item primers were excluded from all following analyses and a refining approach was then used. 
Table 1 Descriptive statistics of all Italian YFAS items

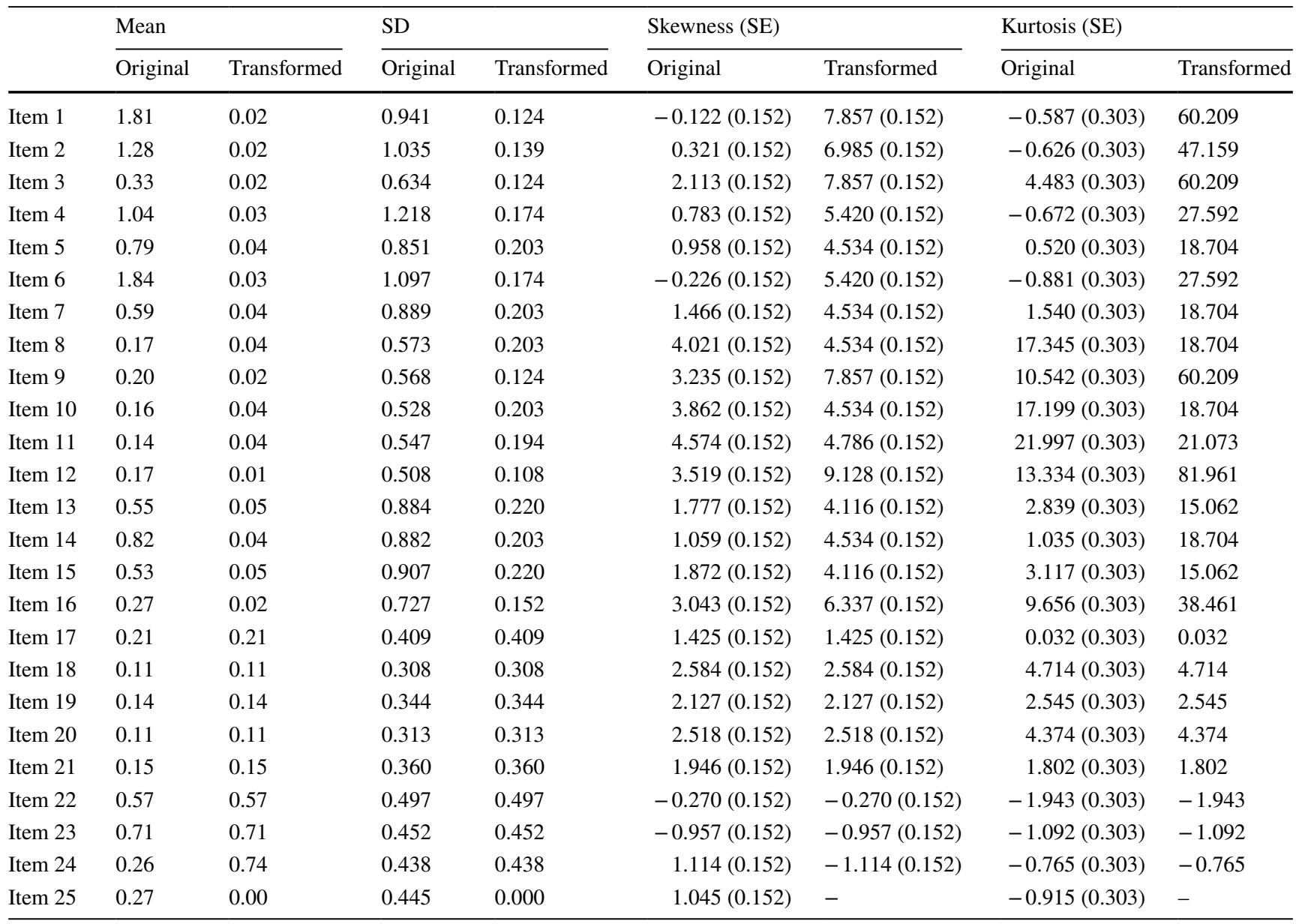

Original original response scale, transformed dichotomization of the item as argued in Gearhardt et al. [4] and Meule and Gearhardt [5]

Given that several items showed high positive skewness and kurtosis values (see Table 1), the Robust Weighted Least Squares estimator (WLSMV) [38, 39] was used to estimate the models' parameters. Factor loadings were tested for statistical significance, while the models' fits to the data were assessed using the following fit indices: (a) the normed $\chi^{2}$ ( $\chi^{2} /$ degrees of freedom), (b) the Comparative Fit Index (CFI), (c) the Root Mean Square Error of Approximation (RMSEA) and (d) the Weight Root Mean square Residual (WRMR) [40]. The normed $\chi^{2}$ is considered an easily, but never formally announced, computed measure of fit [41, 42], and a value of 3 or less is indicative of good fit for dichotomous items [43, 44]. CFI designates the amount of variance and covariance accounted for by the model compared to a baseline model without sample size dependence; values higher than 0.90 are considered good/adequate [45]. The RMSEA expresses fit per degrees of freedom of the model, with values less than 0.08 suggesting an acceptable model fit [45] and values less than 0.05 indicating a good fit [46]. The WRMR measures the (weighted) average differences between the sample and the estimated population variances and covariances; a cut-off value close to 1 is supposed to be good [38]. The factorial structure of the YFAS was further assessed with the seven dichotomous "symptoms" to confirm the conceptual model of food addiction.

Kuder-Richardson's alpha was used to measure the internal consistency of the YFAS with dichotomized items; values equal or higher than 0.7 are indicative of acceptable to high scale reliability [47].

The Pearson product-moment correlation coefficient was used to examine the intercorrelations between the YFAS symptom count and the hypothesized convergent measure. The strength of correlations was interpreted using the Cohen's benchmarks: $<0.10$, trivial; 0.10 to 0.30 , small; 0.30 to 0.50 , moderate; $>0.50$, large. In addition, the Chi square test was performed to assess the associations between the YFAS diagnostic score and the other measures' clinical thresholds.

Mplus 7.0 [38] was used to run all CFAs, while all the other statistical analyses were run with the SPSS software (version 20.0, SPSS Inc., Bologna, Italy). 


\section{Results}

\section{Sample characteristics}

Participants' body mass index (BMI) ranged from 15.6 to 45.9 mean $=21.65 ; \mathrm{SD}=3.06 ;$ Skewness $=2.5(\mathrm{SE}=0.15)$; Kurtosis $=15.78(\mathrm{SE}=0.31)]$. In particular, 216 individuals $(81.2 \%)$ had a BMI that fall into a normal weight range (from 18.5 to 24.9 ) according to the World Health Organization (WHO) BMI classification. Twenty-four participants $(9.8 \%)$ were underweight $(\mathrm{BMI}<18.5)$ and $25(9.8 \%)$ were overweight (BMI $>25)$. For 3 individuals $(1.2 \%)$, it was not possible to compute BMI, because they reported neither weight nor height. The sample BMI was compared with the WHO underweight and overweight cut-off criteria, and one sample's $t$ test revealed that the study sample was neither underweight $(\mathrm{BMI}=18.4 ; t=16.51 ; p<0.001)$ nor overweight $(\mathrm{BMI}=25 ; t=-17.45 ; p<0.001)$ on average.

\section{Structural validity}

The original model including item primers (Model 1) showed an almost acceptable fit to the data (see Table 2). Even if the Chi-square statistic resulted to be statistically significant $\left[\chi^{2}(252)=454.183 ; p<0.001\right]$, the CFI was far from acceptability $(\mathrm{CFI}=0.761)$ and the WRMR value was higher than the suggested cut-off (WRMR $=1.592$ ), both the RMSEA [0.056; 90\% CI 0.048-0.076; $p$ (RMSEA $<0.05)<0.116]$ and the normed $\chi^{2}(1.802)$ were indicative of an adequate fit. As depicted in Table 2, all items' loadings were statistically significant ( $p$ values from 0.006 to $<0.001$ ) and ranged from 0.201 (item \#24) to 0.908 (item \#9 and item\#13) $($ mean $=0.652 ; \mathrm{SD}=0.161)$. Explained variances $\left(R^{2}\right)$ varied from 0.041 (item \#24) to 0.825 (item \#13) (mean $=0.449 ; \mathrm{SD}=0.193$ ).

However, Mplus warned that items \#10 and item \#11 were almost perfectly correlated $(r=0.99)$. An item parcel was thus created by combining item\#10 and item\#11 (this item parcel has a score of 1 when items \#10 or \#11 has a score of 1 and a score of 0 in all other cases). In addition, item\#24 was discarded due to its very low factor loading (0.201).

A second single-factor model (model 2) including the item parcel "item\#10/11" and excluding both the item primers and item\#24 was thus specified and tested. The Chi square statistic resulted to be statistically significant $\left[\chi^{2}(152)=235.690\right.$, $p<0.001]$ and the CFI showed a marginal non-adequate fit (0.858). Also, the WRMR resulted to be higher than the suggested cut-off $(1.236)$, while both the RMSEA [0.046; $90 \%$ CI $0.034-0.058 ; p$ (RMSEA < 0.05$)<0.689]$ and the normed $\chi^{2}(1.556 ;<3)$ were suggestive of a good fit. As reported in Table 2, all items' loadings were statistically significant (all $p$ values $<0.001$ ) and ranged from 0.404 (item \#21) to 0.930 (item \#9) (mean $=0.679 ; \mathrm{SD}=0.156$ ). Explained variances $\left(R^{2}\right)$ varied from 0.163 (item \#21) to 0.865 (item \#9) (mean $=0.485 ; \mathrm{SD}=0.209)$. Internal consistency was acceptable (Kuder-Richardson's alpha $=0.72$ ). Model 2 was thus elected as the best structure for the Italian YFAS in this study and both the symptom counts and the diagnostic scores that were used in the following analyses were computed according to it.

The model including the seven diagnostic symptoms (Model 3) showed an adequate fit to the data. Even if the Chi square statistic was still statistically significant $\left[\chi^{2}\right.$ $(20)=41.911, p=0.003]$, the RMSEA [0.065; 90\% CI 0.037-0.093; $p$ (RMSEA <0.05)<0.166], the CFI $(0.946)$ and the normed $\chi^{2}(2.09)$ revealed a good model fit. The WRMR resulted to be marginally higher than the suggested cut-off (1.132). As depicted in Table 2, all items' loadings were statistically significant (from $p=0.049$ for criterion $\mathrm{B}$ to $p<0.001$ for the other criteria) and ranged from 0.087 (criterion B) to 0.912 (criterion D) $($ mean $=0.679$; $\mathrm{SD}=0.156)$. Explained variance $\left(R^{2}\right)$ varied from 0.008 (criteria $\mathrm{B})$ to 0.832 (criteria $\mathrm{D})($ mean $=0.478 ; \mathrm{SD}=0.289)$.

\section{Convergent validity}

Statistically significant and moderate-to-large correlations were found between the 19-item YFAS symptom counts and the total scores of the BES $(r=0.636 ; p<0.001)$, the EAT-26 $(r=0.534 ; p<0.001)$ and the DEBQ $(r=0.550$; $p<0.001)$. Also, the correlations with the subscales of all questionnaires resulted to be statistically significant (Table 3). Furthermore, the Chi square test showed statistically significant associations between the diagnostic scores and both the BES $\left[\chi^{2}(1)=32.079 ; p<0.001\right]$ and the EAT$26\left[\chi^{2}(1)=13.866 ; p<0.001\right]$ clinical thresholds (the DEBQ scales have no clinical cut-off).

\section{Prevalence of FA symptoms and FA diagnosis according to the italian 19-item YFAS}

The absolute number of FA symptoms that were met by study participants ranges from 0 to 7 (mean $=1.48$; $\mathrm{SD}=1.12$; median $=1$ ). The lowest endorsement rate is for "Substance taken in larger amount and for longer period than intended" (criteria A-4.7\%), while the highest is for "Persistent desire or repeated unsuccessful attempts to quit" (criteria B-84.4\%). The diagnostic threshold for FA was met by $4.3 \%$ of participants $(n=11)$. Three or more symptoms were endorsed by more participants $(n=22 ; 7.7 \%)$, but they did not met the clinical impairment criterion. Descriptive statistics are displayed in Table 4. 
Table 2 Factor loadings, explained variances $\left(R^{2}\right)$ and fit indexes of the three Italian YFAS models

\begin{tabular}{|c|c|c|c|c|c|c|}
\hline & \multicolumn{2}{|l|}{ Model 1} & \multicolumn{2}{|l|}{ Model 2} & \multicolumn{2}{|l|}{ Model 3} \\
\hline & Loadings & $R^{2}$ & Loadings & $R^{2}$ & Loadings & $R^{2}$ \\
\hline Criteria (A) & & & & & 0.853 & 0.727 \\
\hline Item 1 & 0.503 & 0.253 & 0.539 & 0.291 & & \\
\hline Item 2 & 0.645 & 0.416 & 0.663 & 0.440 & & \\
\hline Item 3 & 0.433 & 0.187 & 0.436 & 0.190 & & \\
\hline Criteria (B) & & & & & 0.087 & 0.008 \\
\hline Item 4 & 0.599 & 0.358 & 0.637 & 0.406 & & \\
\hline Item 22 & 0.722 & 0.521 & 0.440 & 0.194 & & \\
\hline Item $24^{\mathrm{a}}$ & 0.201 & 0.041 & - & - & & \\
\hline Item $25^{\mathrm{b}}$ & - & - & - & - & & \\
\hline Criteria (C) & & & & & 0.775 & 0.600 \\
\hline Item 5 & 0.555 & 0.308 & 0.652 & 0.425 & & \\
\hline Item 6 & 0.671 & 0.450 & 0.761 & 0.579 & & \\
\hline Item 7 & 0.473 & 0.223 & 0.597 & 0.357 & & \\
\hline Criteria (D) & & & & & 0.912 & 0.832 \\
\hline Item 8 & 0.767 & 0.588 & 0.830 & 0.689 & & \\
\hline Item 9 & 0.908 & 0.824 & 0.930 & 0.865 & & \\
\hline Item $10^{\mathrm{c}}$ & 0.646 & 0.417 & - & - & & \\
\hline Item $11^{\mathrm{c}}$ & 0.658 & 0.433 & - & - & & \\
\hline Item $10 / 11^{\mathrm{c}}$ & - & - & 0.651 & 0.424 & & \\
\hline Criteria (E) & & & & & 0.438 & 0.192 \\
\hline Item 19 & 0.770 & 0.592 & 0.770 & 0.593 & & \\
\hline Criteria (F) & & & & & 0.630 & 0.397 \\
\hline Item 20 & 0.699 & 0.489 & 0.720 & 0.519 & & \\
\hline Item 21 & 0.458 & 0.210 & 0.404 & 0.163 & & \\
\hline Criteria (G) & & & & & 0.849 & 0.722 \\
\hline Item 12 & 0.681 & 0.464 & 0.854 & 0.730 & & \\
\hline Item 13 & 0.908 & 0.825 & 0.906 & 0.821 & & \\
\hline Item 14 & 0.809 & 0.654 & 0.814 & 0.663 & & \\
\hline Criteria $(\mathrm{H})$ & & & & & 0.591 & 0.349 \\
\hline Item 15 & 0.702 & 0.492 & 0.566 & 0.320 & & \\
\hline Item 16 & 0.704 & 0.496 & 0.740 & 0.547 & & \\
\hline Item primers ${ }^{\mathrm{d}}$ & & & & & - & - \\
\hline Item $17^{\mathrm{d}}$ & 0.819 & 0.671 & - & - & & \\
\hline Item $18^{\mathrm{d}}$ & 0.587 & 0.345 & - & - & & \\
\hline Item $23^{\mathrm{d}}$ & 0.736 & 0.541 & - & - & & \\
\hline$\chi^{2}(d f)$ & $\chi^{2}(252)=454.183^{*}$ & & $\chi^{2}(152)=235.690 *$ & & $\chi^{2}(20)=41.911 * *$ & \\
\hline$\chi^{2} / d f$ & $1.802 ;<2$ & & $1.551 ;<2$ & & $2.09 ;<3$ & \\
\hline RMSEA (90\% CI) & $0.056(0.048 / 0.064)$ & & $0.046(0.034 / 0.058)$ & & $0.065(0.037 / 0.093)$ & \\
\hline$p(\mathrm{RMSEA} \leq 0.05)$ & $p=0.116$ & & $p=0.689$ & & $p=0.166$ & \\
\hline CFI & 0.761 & & 0.858 & & 0.946 & \\
\hline WRMR & 1.592 & & 1.236 & & 1.132 & \\
\hline
\end{tabular}

*Significant for $p<0.001 ; * *$ Significant for $p=0.003$

${ }^{\mathrm{a}}$ Item \#24 has been excluded after Model 1 and because of its starting low estimate standardized factor loading

${ }^{\mathrm{b}}$ Item \#25 was excluded from CFA because of its zero variance (see Table 1)

${ }^{c}$ Due to an almost perfect correlation between item \#10 and \#11 $(r=0.99)$ we created a new item parcel from these items

${ }^{\mathrm{d}}$ Item primer-not scored in the final model 
Table 3 Means, standard deviations and correlations between the Italian 19-item YFAS symptom count and the BES, the EAT26 and the DEBQ scales and subscales

\begin{tabular}{lrll}
\hline & $M$ & SD & YFAS \\
\hline YFAS & 1.54 & 1.24 & - \\
BES Total Score & 8.57 & 6.13 & 0.636 \\
Cognitions/feelings & 3.43 & 2.92 & 0.628 \\
Behaviors & 5.14 & 3.65 & 0.565 \\
EAT.26 total ccore & 11.22 & 8.15 & 0.534 \\
Dieting & 5.46 & 5.83 & 0.512 \\
Bulimia & 3.74 & 1.62 & 0.540 \\
Oral control & 2.02 & 2.45 & 0.200 \\
DEBQ total score & 0.78 & 0.48 & 0.550 \\
Restrained eating & 0.82 & 0.69 & 0.368 \\
Emotional eating & 0.57 & 0.63 & 0.518 \\
External eating & 1.02 & 0.53 & 0.360 \\
\hline
\end{tabular}

All correlations are significant at $p<0.001$

\section{Discussion}

The present study is the first to examine the factorial structure, internal consistency and convergent validity of the Italian version of the YFAS [48] in a multisite sample of postgraduate university students.

Only four previous studies assessed the psychometric properties of the YFAS in non-clinical samples. All of them are first validations of the original [4], German [18], French [20] and Chinese [22] versions. Three out of them used an exploratory approach to assess the YFAS factorial structure $[4,18,20]$ and found support for a single-factor solution. Both Gearhardt et al. [4] and Brunault et al. [20] discarded item\#24 (I have been successful at cutting down or not eating these kinds of food), because of its low factor loading, while Meule et al. [18] decided to retain it and also item\#11 despite their factor loadings resulted to be low as well. Item primers were not included in any factor analysis and only the study of Gearhardt et al. [4] excluded the clinical significance questions.

Only Chen et al. [22] used a confirmatory approach and found that the full-length Chinese YFAS (with the exception of item primers) did not fit their data. Fit indexes did not improve even after removing item\#24 due to its nonsignificant explained variance. They removed also item \#22 and item\#25, because of their low $\mathrm{R}^{2}$ values and the resulting single-factor model showed a better fit to the data. Also, the model including the diagnostic symptoms was tested and showed the best fit indexes [22].

A confirmatory approach was applied also in the present study. The first model (Model 1) included all items (item\#25 was excluded a priori due to its zero variance) and was specified to evaluate the factor loadings of the item primers, which resulted to be medium-to-high. The following model (Model 2) was redefined by excluding the item primers and also item\#24 due to its low factor loading, and by including an item parcel combining item\#10 and item\#11 because of their almost perfect correlation. The last model (Model 3) was specified on the basis of Model 2 but using the seven diagnostic symptoms.

Results showed that Model 2 was the most supported by the CFA fit indexes, which resulted to be acceptable also for Model 3. Item\#22 did not show to be problematic as occurred in Chen et al.'s study [22] and a further difference with their model is the parceling of item\#10 and item\#11. These discrepancies could be due to the cultural and ethnic differences between the two samples, and also to the significant differences in age and BMI. In fact, Chen et al.'s sample had a significantly lower mean age (16.47 vs. 23.8; $p<0.001)$ and the overlap between age ranges is very small (14-19 vs. 18-53). Chen et al.'s sample had also a significantly lower BMI (19.87 vs. 21.65 vs.; $p<0.001)$.

The internal consistency of the Italian 19-item YFAS resulted to be acceptable and all the correlations/associations with the convergent measures, i.e., BES, EAT-26 and

Table 4 Endorsement rates for the Italian 19-item YFAS "symptoms"

\section{Symptom criteria}

NOT met symptom Met symptom criterion criterion

$\begin{array}{rc}244(95.3 \%) & 12(4.7 \%) \\ 40(15.6 \%) & 216(84.4 \%) \\ 233(91.0 \%) & 23(9.0 \%) \\ 236(92.2 \%) & 20(7.8 \%) \\ 221(86.3 \%) & 35(13.7 \%) \\ 202(78.9 \%) & 54(21.1 \%) \\ 238(93.0 \%) & 18(7.0 \%) \\ 240(93.8 \%) & 16(6.3 \%)\end{array}$


DEBQ, were statistically significant and moderate-to-high. This last result is consistent with the first study of Gearhardt et al. [4], who found a significant correlation with the EAT26, the study of Chen et al. [22], who found very similar correlations with the BES and the EAT-26, and also with the study of Brunault et al. [20], who found a comparable non-parametric correlation with the BES.

According to the Italian 19-item YFAS diagnostic scores, $4.3 \%$ of the study participants received a FA diagnosis, which is lower than the prevalence rates found in the other studies on non-clinical samples. Indeed, the prevalence rate was $11.4 \%$ in the study of Gearhardt et al. [4], 9.2\% in the study of Chen et al. [22], $8.8 \%$ in the study of Meule et al. [18] and $8.7 \%$ in the study of Brunault et al. [20]. This difference may depend not only on the peculiar characteristics of the different samples, but may also be due to the different measurement models that were used to compute the YFAS scores.

One important limitation of this study is that some psychometric properties, such as test-retest reliability, discriminant validity and criterion validity, were not assessed. One further limitation is that females are over-represented in the study sample. This prevents the generalization of findings to males and prevented also the assessment of a measurement property that has not been yet assessed in any previous study, that is the YFAS measurement invariance between males and females.

In conclusion, even though a second edition of the YFAS (YFAS 2.0) was recently created in response to the new DSM-5 diagnostic criteria for substance use disorders [49] and its Italian version has been already developed [50], the overlap with the previous DSM-IV criteria [5, 6] enables the Italian 19-item YFAS to be still used for screening purposes both in community health practice and research. For example, it can be applied to the detection of post-graduate university students with FA symptoms to offer them a psychological consultation at the university health center or to address them to an external eating disorders facility. It could also be used as a diagnostic and continuous outcome measure in both clinical practice and clinical research, but only in combination with a clinical interview. Indeed, no study has yet assessed its responsiveness to change and results could thus be misleading.

\section{Compliance with ethical standards}

Conflict of interest On behalf of all authors, the corresponding author states that there is no conflict of interest.

Ethical approval All procedures performed in studies involving human participants were in accordance with the ethical standards of the institutional and/or national research committee and with the 1964 Helsinki declaration and its later amendments or comparable ethical standards.
Informed consent Informed consent was obtained from all participants included in the study prior to accessing the survey.

\section{References}

1. Davis C, Claridge G (1998) The eating disorders as addiction: a psychobiological perspective. Addict Behav 23(4):463-475

2. Grant JE, Potenza MN, Weinstein A, Gorelick DA (2010) Introduction to behavioral addictions. Am J Drug Alcohol Abuse 36(5):233-241. https://doi.org/10.3109/00952990.2010.491884

3. American Psychiatric Association (2013) Diagnostic and statistical manual of mental disorders, 5th edn. American Psychiatric Association, Washington, DC

4. Gearhardt AN, Corbin WR, Brownell KD (2009) Preliminary validation of the Yale Food Addiction Scale. Appetite 52(2):430-436. https://doi.org/10.1016/j.appet.2008.12.003

5. Meule A, Gearhardt AN (2014) Food addiction in the light of DSM-5. Nutrients 6(9):3653-3671. https://doi.org/10.3390/nu609 3653

6. Meule A, Gearhardt AN (2014) Five years of the Yale Food Addiction Scale: taking stock and moving forward. Curr Addict Rep 1(3):193-205. https://doi.org/10.1007/s40429-014-0021-z

7. Pursey KM, Stanwell P, Gearhardt AN, Collins CE, Burrows TL (2014) The prevalence of food addiction as assessed by the Yale Food Addiction Scale: a systematic review. Nutrients 6(10):45524590. https://doi.org/10.3390/nu6104552

8. Brownell KD, Gold MS (2013) Food and addiction: scientific, social, legal, and legislative implications. In: Food and addiction. Oxford University Press, Oxford, pp 439-446

9. Tonioni F, Mazza M, Autullo G, Cappelluti R, Catalano V, Marano G, Fiumana V, Moschetti C, Alimonti F, Luciani M, Lai C (2014) Is internet addiction a psychopathological condition distinct from pathological gambling? Addict Behav 39(6):1052-1056. https:// doi.org/10.1016/j.addbeh.2014.02.016

10. Avena NM, Rada P, Hoebel BG (2008) Evidence for sugar addiction: behavioral and neurochemical effects of intermittent, excessive sugar intake. Neurosci Biobehav Rev 32(1):20-39. https:// doi.org/10.1016/j.neubiorev.2007.04.019

11. Hebebrand J, Albayrak O, Adan R, Antel J, Dieguez C, de Jong J, Leng G, Menzies J, Mercer JG, Murphy M, van der Plasse G, Dickson SL (2014) "Eating addiction", rather than "food addiction", better captures addictive-like eating behavior. Neurosci Biobehav Rev 47:295-306. https://doi.org/10.1016/j.neubi orev.2014.08.016

12. Hone-Blanchet A, Fecteau S (2014) Overlap of food addiction and substance use disorders definitions: analysis of animal and human studies. Neuropharmacology 85:81-90. https://doi.org/10.1016/j. neuropharm.2014.05.019

13. Volkow ND, Wang GJ, Fowler JS, Telang F (2008) Overlapping neuronal circuits in addiction and obesity: evidence of systems pathology. Philos Trans R Soc Lond Ser B Biol Sci 363(1507):3191-3200. https://doi.org/10.1098/rstb.2008.0107

14. Wang GJ, Volkow ND, Thanos PK, Fowler JS (2009) Imaging of brain dopamine pathways: implications for understanding obesity. J Addict Med 3(1):8-18. https://doi.org/10.1097/ADM.0b013 e31819a86f7

15. Volkow ND, Wang GJ, Tomasi D, Baler RD (2013) Obesity and addiction: neurobiological overlaps. Obes Rev 14(1):2-18. https ://doi.org/10.1111/j.1467-789X.2012.01031.x

16. Gearhardt AN, Yokum S, Orr PT, Stice E, Corbin WR, Brownell KD (2011) Neural correlates of food addiction. Arch Gen Psychiatry 68(8):808-816. https://doi.org/10.1001/archgenpsychiat ry. 2011.32 
17. Gearhardt AN, White MA, Masheb RM, Morgan PT, Crosby RD, Grilo CM (2012) An examination of the food addiction construct in obese patients with binge eating disorder. Int J Eat Disord 45(5):657-663. https://doi.org/10.1002/eat.20957

18. Meule A, Vögele C, Kübler A (2012) German translation and validation of the Yale Food Addiction Scale. Diagnostica 3(12):115-126

19. Meule A, Heckel D, Kubler A (2012) Factor structure and item analysis of the Yale Food Addiction Scale in obese candidates for bariatric surgery. Eur Eat Disord Rev J Eat Disord Assoc 20(5):419-422. https://doi.org/10.1002/erv.2189

20. Brunault P, Ballon N, Gaillard P, Reveillere C, Courtois R (2014) Validation of the French version of the yale food addiction scale: an examination of its factor structure, reliability, and construct validity in a nonclinical sample. Can J Psychiatry Revue canadienne de psychiatrie 59(5):276-284

21. Granero R, Hilker I, Aguera Z, Jimenez-Murcia S, Sauchelli S, Islam MA, Fagundo AB, Sanchez I, Riesco N, Dieguez C, Soriano J, Salcedo-Sanchez C, Casanueva FF, De la Torre R, Menchon JM, Gearhardt AN, Fernandez-Aranda F (2014) Food addiction in a Spanish sample of eating disorders: DSM-5 diagnostic subtype differentiation and validation data. Eur Eat Disord Rev J Eat Disord Assoc 22(6):389-396. https://doi.org/10.1002/erv.2311

22. Chen G, Tang Z, Guo G, Liu X, Xiao S (2015) The Chinese version of the Yale Food Addiction Scale: an examination of its validation in a sample of female adolescents. Eat Behav 18:97-102. https://doi.org/10.1016/j.eatbeh.2015.05.002

23. Innamorati M, Imperatori C, Manzoni GM, Lamis DA, Castelnuovo G, Tamburello A, Tamburello S, Fabbricatore M (2015) Psychometric properties of the Italian Yale Food Addiction Scale in overweight and obese patients. Eat Weight Disord EWD 20(1):119-127. https://doi.org/10.1007/s40519-014-0142-3

24. First MB, Spitzer RL, Gibbon M, Williams JB (2002) Structured clinical interview for DSM-IV-TR axis I disorders, research version, patient edition, SCID-I/P. In: Institute NYSP (ed) Biometrics research. American Psychiatric Press Inc, New York

25. Gormally J, Black S, Daston S, Rardin D (1982) The assessment of binge eating severity among obese persons. Addict Behav $7(1): 47-55$

26. Ricca $V$ et al (2000) Screening for binge eating disorder in obese outpatients. Compr Psychiatry 41(2):111-115

27. Marcus MD, Wing RR, Hopkins J (1988) Obese binge eaters: affect, cognitions, and response to behavioural weight control. J Consult Clin Psychol 56(3):433-439

28. Hood MM, Grupski AE, Hall BJ, Ivan I, Corsica J (2013) Factor structure and predictive utility of the Binge Eating Scale in bariatric surgery candidates. Surg Obes Relat Dis 9(6):942-948. https://doi.org/10.1016/j.soard.2012.06.013

29. Marek RJ, Tarescavage AM, Ben-Porath YS, Ashton K, Heinberg LJ (2015) Replication and evaluation of a proposed two-factor Binge Eating Scale (BES) structure in a sample of bariatric surgery candidates. Surg Obes Relat Dis 11(3):659-665. https://doi. org/10.1016/j.soard.2014.09.015

30. Robert SA, Rohana AG, Suehazlyn Z, Maniam T, Azhar SS, Azmi KN (2013) The validation of the malay version of binge eating scale: a comparison with the structured clinical interview for the DSM-IV. J Eat Disord 1:28. https://doi. org/10.1186/2050-2974-1-28

31. Marek RJ, Ben-Porath YS, Ashton K, Heinberg LJ (2014) Impact of using DSM-5 criteria for diagnosing binge eating disorder in bariatric surgery candidates: change in prevalence rate, demographic characteristics, and scores on the Minnesota Multiphasic
Personality Inventory-2 restructured form (MMPI-2-RF). Int J Eat Disord 47(5):553-557. https://doi.org/10.1002/eat.22268

32. Garner DM, Olmsted MP, Bohr Y, Garfinkel PE (1982) The eating attitudes test. Psychometric features and clinical correlates. Psychol Med 12(4):871-878

33. Dotti A, Lazzari R (1998) Validation and reliability of the Italian EAT-26. Eat Weight Disord 3(4):188-194

34. Van Strien T, Frijters JER, Bergers GPA, Defares PB (1986) The Dutch Eating Behaviour Questionnaire (DEBQ) for assessment of restrained, emotional and external eating behaviour. Int J Eat Disord 5:747-755

35. Dakanalis A, Zanetti MA, Clerici M, Madeddu F, Riva G, Caccialanza R (2013) Italian version of the Dutch Eating Behavior Questionnaire. Psychometric proprieties and measurement invariance across sex, BMI-status and age. Appetite 71:187-195. https ://doi.org/10.1016/j.appet.2013.08.010

36. Riva G, Molinari E (2004) Clinical psychology of obesity. Bollati Boringhieri, Turin

37. Van Strien T, Herman CP, Verheijden MW (2013) Eating style, overeating and weight gain. A prospective 2-year follow-up study in a representative Dutch sample. Appetite 59(3):782-789

38. Muthén LK, Muthén BO (1998-2012) Mplus User's Guide. 7th edn. Muthén \& Muthén, Los Angeles

39. Muthén B, du Toit SHC, Spisic D (1997) Robust inference using weighted least squares and quadratic estimating equations in latent variable modeling with categorical and continuous outcomes. Psychometrika 75(1):40-45

40. Barrett $P$ (2007) Structural equation modelling: adjudging model fit. Personal Individ Differ 42(5):815-824

41. Hoyle RH (2012) Handbook of strucural equation modeling. The Guildford Press, New York

42. Marsh HW, Hocevar D (1985) Application of confirmatory factor analysis to the study of self-concept: first-and higher order factor models and their invariance across groups. Psychol Bull 97(3):562-582

43. Wheaton B, Muthén B, Alwin D, Summers G (1977) Assessing reliability and stability in panel models. In: Heise DR (ed) Sociological methodology. Jossey-Bass, Inc., San Francisco, pp 84-136

44. Tay L, Drasgow F (2012) Adjusting the adjusted $\chi 2 / d f$ ratio statistic for dichotomous item response theory analyses does the model fit? Educ Psychol Meas 72(3):510-528

45. Hu LT, Bentler PM (1999) Cutoff criteria for fit indexes in covariance structure analysis: conventional criteria versus new alternatives. Struct Equ Model 6:1-55

46. Browne MW, Cudeck R (1990) Single sample cross-validation indices for covariance structures. Multivar Behav Res 24:445-455

47. Kuder GF, Richardson MW (1937) The theory of the estimation of test reliability. Psychometrika 2:151-160

48. Innamorati $\mathrm{M}$, Imperatori $\mathrm{C}$, Meule $\mathrm{A}$, Lamis DA, Contardi A, Balsamo M, Tamburello A, Tamburello S, Fabbricatore M (2015) Psychometric properties of the Italian Food Cravings Questionnaire-Trait-reduced (FCQ-T-r). Eat Weight Disord EWD 20(1):129-135. https://doi.org/10.1007/s40519-014-0143-2

49. Gearhardt AN, Corbin WR, Brownell KD (2016) Development of the Yale Food Addiction Scale Version 2.0. Psychol Addict Behav J Soc Psychol Addict Behav 30(1):113-121. https://doi. org/10.1037/adb0000136

50. Aloi M, Rania M, Rodriguez Munoz RC, Jimenez Murcia S, Fernandez-Aranda F, De Fazio P, Segura-Garcia C (2017) Validation of the Italian version of the Yale Food Addiction Scale 2.0 (I-YFAS 2.0) in a sample of undergraduate students. Eat Weight Disord EWD. https://doi.org/10.1007/s40519-017-0421-x 


\section{Affiliations}

\section{Gian Mauro Manzoni ${ }^{1,2}$ - Alessandro Rossi ${ }^{1,4}$. Giada Pietrabissa ${ }^{1,3}$. Giorgia Varallo ${ }^{1}$ Enrico Molinari ${ }^{1,3}$. Eleonora Poggiogalle ${ }^{5}$. Lorenzo Maria Donini ${ }^{5}$. Giulietta Tarrini ${ }^{6} \cdot$ Nazario Melchionda $^{6}$. Carla Piccione ${ }^{7}$. Giovanni Gravina $^{8}$. Gianluigi Luxardi ${ }^{9}$. Emilia Manzato ${ }^{10} \cdot$ Romana Schumann $^{11}$ - Marco Innamorati ${ }^{12}$. Claudio Imperatori ${ }^{12} \cdot$ Mariantonietta Fabbricatore $^{12}$. Gianluca Castelnuovo ${ }^{1,3}$}

1 Istituto Auxologico Italiano IRCCS, Psychology Research Laboratory, Via Luigi Cadorna, 90, Piancavallo, Oggebbio, VCO, Italy

2 Faculty of Psychology, eCampus University, Novedrate, Como, Italy

3 Department of Psychology, Catholic University, Milan, Italy

4 Interdepartmental Center for Family Research, Department of Philosophy, Sociology, Education, and Applied Psychology, Section of Applied Psychology, University of Padova, Padua, Italy

5 Department of Experimental Medicine, Sapienza University, Rome, Italy

6 Department of Internal Medicine, University Alma mater, Bologna, Italy
7 Center for Eating Disorders, S. Rossore Clinic, Pisa, Italy

8 Center for Eating Disorders, ASL Tuscany Nord West, Pisa, Italy

9 Center for Eating Disorders, ASL5 West Friuli, Pordenone, Italy

10 Eating and Weight Disorders Centre, Private Hospital Salus, Ferrara, Italy

11 Gruber Center for Eating Disorders, Bologna, Italy

12 Department of Human Sciences, European University of Rome, Rome, Italy 